nachteilig für alleinstehende Frauen und Frauen in einer homosexuellen Partnerschaft aus, die regelmäßig diese medizinischen Erfordernisse nicht erfüllen ${ }^{61}$. Die Richtliniengebung der Bundesärztekammer ist als hoheitliche Tätigkeit anzusehen. Insoweit ist $\mathrm{zu}$ beachten, dass sich eine Indikationsstellung im Rahmen einer hoheitlichen Tätigkeit nach dem modernen Eingriffsbegriff an den Grundrechten messen lassen muss ${ }^{62}$. Eine hoheitliche Beschränkung des Zugangs zu Maßnahmen der assistierten Reproduktion bedarf insoweit einer Rechtfertigung der damit verbundenen Einschränkung des Grundrechts auf freie Reproduktion. Nach dem Ergebnis der Diskussion um die statusrechtlichen Vorgaben der bisherigen (Muster-) Richtlinie ist eine Rechtfertigung dieses Eingriffs nicht ersichtlich ${ }^{63}$. Auch stellt bezüglich der Richtliniengebung nach $\$ 16 \mathrm{~b}$ TPG die Frage, ob die Bundesärztekammer hinreichend personell legitimiert ist ${ }^{64}$.

\section{Fazit}

Es ist durchweg zu begrüßen, dass die Bundesärztekammer in jüngerer Zeit mit der (Muster-) Richtlinie zur assistierten Reproduktion und dem Verbot ärztlicher Suizidbeihilfe zwei verfassungswidrige Vorschriften aus der Musterberufsordnung gestrichen hat. Es ist nun an der Zeit, Lehren aus der jahrelangen Diskussion und der Rüge durch das BVerfG zu ziehen und diese Lehren zukünftig auch konsequent umzusetzen.

61) Krekeler, Berufsordnungen im Rahmen der Verfassung, 2021, S. $92 \mathrm{f}$.

62) Krekeler, Berufsordnungen im Rahmen der Verfassung, 2021, S. $413 \mathrm{ff}$.

63) Vgl. dazu Krekeler, Berufsordnungen im Rahmen der Verfassung, 2021, S. $425 \mathrm{ff}$.

64) Vgl. dazu Krekeler, Berufsordnungen im Rahmen der Verfassung, 2021, S. 420 ff. m.w. N.

\title{
Rechtsprechungsübersicht zum Medizinprodukterecht und zu angrenzenden Gebieten 2020/2021
}

\section{Sandra Hobusch und Sebastian Ochs}

\section{Vorbemerkungen}

Die nachfolgende Rechtsprechungsübersicht knüpft an den Vorjahresbericht von Hobusch/Ochs, MedR 2020, $911 \mathrm{ff.}$ an. Sie gibt erneut einen Überblick über Medizinprodukte in der Rechtsprechung zum Medizinprodukte-, Wettbewerbs-, Haftungs- und Krankenversicherungsrecht.

\section{Medizinprodukterecht}

a) Begriff und Einstufung

Nach $₫ 3$ Nr. 1 MPG (bzw. auch Art. 2 Nr. 1 MDR) kann es sich bei Software um ein Medizinprodukt handeln. Im Rahmen eines wettbewerbsrechtlichen Verfahrens vor dem LG Hamburg ${ }^{1}$ war die Einordnung einer ohne CE-Kennzeichnung vertriebenen Software streitig, die bei der Dokumentation der Aufbereitung medizinischer Instrumente eingesetzt wird. Die Software ist nicht in einem Desinfektionsgerät integriert, sondern wird separat von den bei der Aufbereitung verwendeten Geräten vertrieben. Das LG sah in der Software weder eine eigenständige noch eine Steuerungssoftware i.S. des $\$ 3$ Nr. 1 MPG. Nach Ansicht des LG fehlt es an den in $\$ 3 \mathrm{Nr}$. 1 MPG beschriebenen Zwecken. Die Software bestimme nicht die diagnostische oder therapeutische Hauptfunktion des eingesetzten Desinfektionsgeräts. Sie erhebe und analysiere die Daten auch nicht eigenständig. Dies erfolge durch das bei der Aufbereitung eingesetzte Gerät selbst. Zweck der Software sei es nur, die Daten auszulesen und für den Anwender lesbar zu machen. Die streitige Software nehme keine eigenen Berechnungen

Prof. Dr. iur. Sandra Hobusch,

Ostfalia Hochschule für angewandte Wissenschaften,

Rothenfelder Straße 10, 38440 Wolfsburg, Deutschland

Rechtsanwalt Sebastian Ochs,

Grünecker Patent- \& Rechtsanwälte,

Leopoldstraße 4, 80802 München, Deutschland vor und gebe in den Protokollen keine eigenen bewertenden Hinweise, wie z.B. ,sterile Beladung“, ab, sondern übernehme nur die Informationen, die das Desinfektionsgerät erstellt.

Der Bay. VGH ${ }^{2}$ stufte wie die Vorinstanz Wurzelkanalinstrumente eines Zahnarztes als „kritisch B“ ein. Er führte in dem Zusammenhang aus, dass nach dem von der Vorinstanz eingeholten Sachverständigengutachten und dem Hygieneplan der BZÄK zwar grundsätzlich die Einstufung als ,kritisch A“ und ,kritisch B“" in Betracht komme. Wenn jedoch die Effektivität der Reinigung durch Inspektion nicht unmittelbar beurteilbar sei, im zu entscheidenden Fall wegen der fehlenden Lichtlupe, so seien die Instrumente als „kritisch B“ einzustufen.

Das OLG Hamm ${ }^{3}$ entschied im Rahmen einer wettbewerbsrechtlichen Streitigkeit über die Frage, ob eine sogenannte „Alltagsmaske“ in Form einer Stoffmaske als Mund-Nase-Bedeckung ein Medizinprodukt ist und welche Konsequenzen dies hat. Der Senat stufte die Stoffmaske nicht als Medizinprodukt ein. Insoweit komme es für die Beurteilung auf die subjektive Bestimmung des Herstellers an. Maßgeblich sei, was der Verkehr der Kennzeichnung des Produkts, seiner Gebrauchsanweisung sowie der Bewerbung entnehme. Ein ausdrücklicher Hinweis auf die Verwendbarkeit zu medizinischen Zwecken fehlte bei der Alltagsmaske selbst sowie auf deren Verpackung. Auch aus der Gestaltung und Aufmachung ergab sich nichts Abweichendes. Vielmehr spreche nach dem OLG die comicartige Darstellung auf der Alltagsmaske dagegen, dass der angesprochene Verkehr diese als für medizinische Zwecke verwendbar erkenne. Irrelevant sei, dass die Alltagsmaske im Einzelhandel auch neben ,medizinisch anmutenden Gesichtsmasken“" angeboten werde. Genauso wenig komme es

1) LG Hamburg, Urt. v. 12.8.2020 - 416 HKO 114720 -, juris; Berufung anhängig: OLG Hamburg, 15 U 179/20.

2) Bay. VGH, Beschl. v. 3.3.2021 - 22 ZB 20.1685 -, juris.

3) OLG Hamm, Beschl. v. 15.12.2020 - $4 \mathrm{~W} \mathrm{116/20-,} \mathrm{GRUR-RR}$ 2021, $92 \mathrm{ff}$. 
darauf an, ob der Alltagsmaske tatsächlich eine Schutzwirkung gegen das Coronavirus SARS-CoV-2 zugesprochen werde. Aus wettbewerbsrechtlicher Sicht bedürfe es keines Hinweises darauf, dass es sich nicht um ein Medizinprodukt handelt. Daher stelle der Vertrieb ohne einen solchen Hinweis nach dem OLG Hamm keine irreführende Werbung nach $₫ 5, \S 5$ a UWG, $₫ 3 \mathrm{HWG}$ dar.

Die Frage der Einstufung eines Produkts als Medizinprodukt war auch Gegenstand eines wettbewerbsrechtlichen Verfahrens vor dem OLG Köln ${ }^{4}$. Ausgangsgrundlage für das Verfahren war der Vertrieb des Produkts „Femanno$\mathrm{se}^{\circledR \text { ¿ }}$ mit den wesentlichen Wirkstoffen D-Mannose und Cranberry-Extrakt zur Behandlung von Blasenentzündung und Harnwegsinfekten als Medizinprodukt. Das OLG stellte fest, dass es sich bei den Produkten um ein Arzneimittel handele, weil die Hauptwirkung der Produkte eine pharmakologische und keine lediglich physikalische sei. Der Vertrieb als Medizinprodukt verstieß nach dem Senat damit gegen $\$ \int 3,3 \mathrm{a}, 5$ Abs. 1 S. 1 Nr. 1 UWG, $\$ 3$ MPG, S\$ 3 , 3a HWG, $\$ 21$ AMG.

Und auch das OLG Frankfurt a. M. ${ }^{5}$ hatte sich zur Frage der Einstufung eines Produkts als Arzneimittel oder Medizinprodukt zu verhalten. Streitig war die Frage, ob der Hustensaft der Beklagten ein verschreibungspflichtiges Arzneimittel ist oder aber ein Medizinprodukt, als welches dieser auch vertrieben wurde. Das Bundesinstitut für Arzneimittel und Medizinprodukte hatte zu dem Hustensaft einen Bescheid erlassen, wonach es sich dabei nicht um ein zulassungspflichtiges Arzneimittel nach $\$ 2$ Abs. 1 AMG handele, sondern um ein Medizinprodukt nach $\$ 3 \mathrm{Nr} .1$ MPG. Die Beklagte berief sich auf diesen Bescheid und eine hierdurch vermittelte Tatbestandswirkung. Der Senat kam abweichend zu dem Ergebnis, dass ein Arzneimittel vorliege. Der Bescheid entfalte auch keine Tatbestandswirkung, die dem entgegenstehe. Zwar könne ein Verwaltungsakt, der ein bestimmtes Marktverhalten ausdrücklich erlaube, ein Verhalten als rechtmäßig qualifizieren. Der Bescheid des Bundesinstituts für Arzneimittel und Medizinprodukte stelle auch einen Verwaltungsakt dar, der rechtsgestaltende Wirkung habe und könnte daher auch eine Tatbestandswirkung auslösen. Ob der Verwaltungsakt rechtmäßig ergangen ist, sei zudem nicht zu prüfen, wobei die Nichtigkeit nach $\$ 44$ Abs. $1 \mathrm{VwVfG}$ wegen eines schwerwiegenden Fehlers die Grenze darstelle. Es komme aber für die Frage der Tatbestandswirkung auch maßgeblich auf die Begründung des Verwaltungsakts an. Die Beklagte hatte den Bescheid nur teilweise geschwärzt vorgelegt. Der Hinweis der Beklagten auf schutzwürdige Geschäftsgeheimnisse, die in der Begründung genannt seien, verhalf ihr ebenfalls nicht. Der Senat verwies auf die einschlägigen Schutzmaßnahmen gemäß $\delta \int 172$ Nr. 2, 174 Nr. 3 GVG. Der Senat hielt fest, dass er mangels Vorlage des ungeschwärzten Bescheides dessen Regelungsgehalt nicht vollständig nachvollziehen könne, was zulasten der Beklagten gehe. Insbesondere könne nicht erkannt werden, ob der Bescheid sich auch auf Präsentationsarzneimittel beziehe und insoweit die Tatbestandswirkung auch greife. In seiner weiteren Begründung hielt das OLG dann fest, dass es sich bei dem Hustensaft um kein Medizinprodukt handele, sondern um ein Präsentationsarzneimittel. Insoweit berücksichtigte der Senat auch, dass weitere Produkte der Beklagten als Arzneimittel vertrieben werden.

\section{b) Abgabe von Medizinprodukten}

Nach $₫ 3$ Abs. 4 MPAV dürfen In-vitro-Diagnostika, die für den direkten oder indirekten Nachweis eines Erregers für die Feststellung bestimmter Infektionskrankheiten (u. a. COVID-19) nur an Ärzte, ambulante und stationäre Einrichtungen im Gesundheitswesen, Großhandel und Apotheken, an Gesundheitsbehörden des Bundes, der Länder, der Gemeinden und Gemeindeverbände, Blutspendedienste, pharmazeutische Unternehmen, Beratungs- und Testeinrichtungen für besonders gefährdete Personengruppen abgegeben werden. Davon ausgenommen sind die in Anlage 3 aufgeführten In-vitro-Diagnostika. Durch die 3 . VO zur Änderung der MPAV v. 1.2.2021 ${ }^{6}$ sind die In-vitroDiagnostika für die Eigenanwendung, die für den direkten Erregernachweis des Coronavirus SARS-CoV-2 bestimmt sind, in die Anlage 3 aufgenommen worden. Im November 2020 musste sich das LG Hamburg ${ }^{7}$ im Rahmen einer wettbewerbsrechtlichen Streitigkeit mit dem sog. Coronavirus PCR Test Kit befassen, das die Antragsgegnerin gegenüber Verbrauchern bewarb. Das LG kam zwar zu dem Ergebnis, dass der Antragsgegnerin die Bewerbung des Test Kits wegen eines Verstoßes gegen $\$ \$ 3,3 a$ UWG i.V.m. $\$ 12$ HWG zu untersagen sei. Einen Verstoß gegen das Abgabeverbot gem. $\$ 3$ Abs. 4 MPAV konnte das LG dagegen nicht feststellen. Bei dem Test Kit handelte es sich um ein Probenahme- und Einsendeset, das aus einem oder mehreren gebrauchsfertigen Rachenabstrichspateln und Probenröhrchen zur Aufnahme der Abstrichspatel sowie einer Versandverpackung bestand. Die Analyse der Probe, die die Antragsgegnerin zusammen mit dem Test Kit offerierte, erfolgte in einem Labor. Folglich kam dem Test Kit nur die Zweckbestimmung zu, das durch den Rachenabstrich gewonnene Zellmaterial aufzubewahren und den Versand $\mathrm{zu}$ ermöglichen. Das in $\$ 3$ Abs. 4 MPAV geregelte Abgabeverbot an Verbraucher setze jedoch voraus, so das LG nach Auslegung der Vorschrift, dass das Produkt dazu bestimmt sei, durch Eigenanwendung des medizinischen Laien den Nachweis des Krankheitserregers zu erbringen.

\section{Medizinprodukte und Wettbewerbsrecht}

\section{a) Irreführende Werbung}

Wird ein Hustensaft mit dem Hinweis, dass dieser auch gegen produktiven Husten helfe, wie bspw. in ,,ist für die Behandlung von trockenem und produktiven Husten [...] geeignet", liegt darin eine irreführende Angabe, wenn die beworbene therapeutische Wirkung nicht besteht, so das OLG Karlsruhe ${ }^{8}$. Diese Form der Bewerbung beinhalte die Behauptung einer vorbehaltlosen und uneingeschränkten Wirksamkeit gegen produktiven Husten. Aufgrund der Nennung von trockenem und produktivem Husten gehe der Durchschnittsverbraucher davon aus, dass das Produkt auch gegen beide Arten wirke. Eine Einschränkung auf bestimmte Formen produktiven Hustens erkannte das OLG in der Angabe, dass das Produkt auf die oberen Atemwege wirke, nicht. Es fehle an einer nötigen, für den Durchschnittsverbraucher hinreichend deutlichen Bezugnahme auf die beworbene Wirksamkeit gegen produktiven Husten. Der Senat beschäftigte sich auch mit der Frage, ob die CE-Zertifizierung eine Sperrwirkung entfalte, die der Werbung die Unlauterkeit nehmen könnte. Diesen Punkt ließ das OLG aber im Ergebnis offen, weil schon nicht nachgewiesen werden konnte, dass alle Herstellerangaben von der Benannten Stelle, mithin auch die Wirksamkeitsangaben, geprüft und bescheinigt wurden. Die Beklagte hatte sich im Rahmen der Zertifizierung nämlich für ein

4) OLG Köln, Urt. v. 23.12.2020 - 6 U 18/20 -, PharmR 2021, $144 \mathrm{ff}$.

5) OLG Frankfurt a. M., Urt v. 22.5.2020 - 6 U 23/20 -, GRURRR 2020, 456ff.

6) Dritte Verordnung zur Änderung der Medizinprodukte-Abgabeverordnung im Rahmen der epidemischen Lage von nationaler Tragweite v. 1.2.2021, BAnz AT 2.2.2021 V1.

7) LG Hamburg, Urt. v. 17. 11.2020 - $416 \mathrm{HKO} 144 / 20$-, juris

8) OLG Karlsruhe, Urt. v. 14.10.2020 - 6 U 59/20 -, GRUR-RS 2020, 37076 
Verfahren entschieden, bei dem die Wirksamkeit des Produkts nicht geprüft und bescheinigt wird. Das OLG Karlsruhe stufte die Bewerbung als unzulässig, weil irreführend nach $\$ \$ 3$, 3a UWG, $\$ 3$ S. 1, S. 2 Nr. 1 HWG, ein.

\section{b) Werbung mit Werbegaben gem. \ 7 HWG}

Eine Süßigkeitenbox im Wert von circa 5 Euro als kostenlose Beigabe für den Bezug von Mullbinden und Mullkompressen stellt eine unzulässige Werbegabe nach $\$ 7$ Abs. 1 HWG dar, so das OLG Hamm. Die Beklagte bewarb ihre Produkte gegenüber Apotheken damit, bei einem Bestellwert von 30 Euro kostenlos eine Süßigkeitenbox zuzugeben. Der Begriff der „Werbegabe“ sei weit auszulegen. $\$ 7$ Abs. $1 \mathrm{HWG}$ ziele auf eine weitgehende Eindämmung von Werbegeschenken im Heilmittelbereich ab. Denn davon gehe eine abstrakte Gefahr einer unsachlichen Beeinflussung aus. Die Werbung sei hier direkt auf die Apotheker gerichtet. Für diese sei auch erkennbar, dass sie und nicht ihre Kunden angesprochen seien. Mithin qualifizierte das OLG die Boxen als Werbegaben und nicht als Werbehilfen. Mit einem Wert von circa 5 Euro liege keine geringwertige Kleinigkeit im Sinne von $\$ 7$ Abs. 1 S. 1 Nr. $1 \mathrm{HWG}$ vor. Die Frage der Geringwertigkeit dürfe nicht in Abhängigkeit von dem Warenwert bestimmt werden, so der Senat. Selbst wenn man aber eine Geringwertigkeit annehmen wollte, fehlte nach dem OLG Hamm die erforderliche Bestimmung zur Verwendung in der pharmazeutischen Praxis gemäß $\int 7$ Abs. 1 S. 2 HWG. Dabei gelte ein objektiver Maßstab, wobei denkbare andere Eignungen zur Verwendung außen vor bleiben müssen. Eine rein private Nutzung durch die Apotheker sei denkbar, und damit auch eine Verwendung außerhalb der pharmazeutischen Praxis. Für die Beklagte stritt somit keiner der Ausnahmetatbestände.

Die Werbung „Wir schenken Ihnen eine neue Brille. Inklusive Gläsern. Große X Geschenkaktion für unsere Helden. Exklusiv für Pflegerinnen, Pfleger, Ärztinnen und Ärzte.“ für Brillen, bei denen die angesprochenen Verkehrskreise aus mehreren Kollektionen wählen können, ist unzulässig nach $₫ 7$ Abs. $1 \mathrm{~S}$. $1 \mathrm{HWG}$, so das OLG Stuttgart ${ }^{10}$. In der kostenlosen Abgabe einer Brille liege das Angebot einer unzulässigen Werbegabe. Dabei könne auch eine Werbung für das gesamte Warensortiment eine produktbezogene Werbung darstellen. Es handele sich dann immer noch um Absatzwerbung, und nicht um Firmenwerbung. Die Darstellung der Aktion als „,Dankesaktion für Corona-Helden“ ändere daran nichts. Anders als noch die Vorinstanz sah das OLG in der Werbung auch eine abstrakte Gefahr einer unsachlichen Beeinflussung begründet. Das Erfordernis der unsachlichen Beeinflussung setze schon keine unmittelbare Kopplung zwischen dem Erhalt der Werbegabe und einer Kaufentscheidung voraus. $\mathrm{Ob}$ die Gefahr einer unsachlichen Beeinflussung vorliegt, sei im Sinne einer individuellen Beeinflussbarkeit der Zuwendungsempfänger zu bewerten. Insoweit bestehe das Risiko, dass der Zuwendungsempfänger bei Abholung des Geschenks weitere Produkte erwerbe. Nach dem OLG konnte sich die Werbende auch nicht auf einen der Ausnahmetatbestände berufen. Die Abgabe einer kostenlosen Brille stelle keinen hundertprozentigen Geldrabatt dar. Ein Berufen auf $₫ 7$ Abs. 1 Nr. 2 lit. a) HWG sei der Beklagten damit untersagt. Andernfalls würde dies, so der Senat, zu einer Aushöhlung von $₫ 7$ Abs. $1 \mathrm{HWG}$ führen. Mangels Erwerbsgeschäft greife auch der Ausnahmetatbestand des Naturalrabatts nach $₫ 7$ Abs. S. 1 Nr. 2 lit. b) HWG nicht.

Zahnbürsten seien Bedarfsgegenstände und Werbung für Zahnbürsten sei keine krankheitsbezogene Werbung, so das OLG Hamburg ${ }^{11}$. Das HWG finde daher darauf keine Anwendung. Wirbt ein Unternehmen für den Kauf seiner Zahnbürsten mit einer Beteiligung an den Kosten für eine Zahnreinigung oder Zahnaufhellung, stelle dies keine un- zulässige Werbegabe an die Zahnärzte nach $₫ 7$ Abs. 1 S. 1 HWG dar. Soweit die Patienten betroffen sind, sei die $\mathrm{Zu}-$ wendung zulässig nach $\$ 7 \mathrm{Abs} .1 \mathrm{Nr}$. 2 lit. a) HWG, so der Senat. Die Klägerin störte sich an der Bewerbung der Beklagten für deren Zahnbürsten mittels Flyern, die die Beklagte an Zahnärzte zur Auslage in den Praxisräumen verteilte. In den Flyern wurde neben einem Rabatt von $30 \%$ auf den Kauf einer der Zahnbürsten eine Beteiligung an den Kosten für eine Zahnreinigung oder Zahnaufhellung ausgelobt. Das OLG Hamburg stufte die Werbung als zulässig ein. Eine Zuwendung an die Ärzte scheide aus, weil diese auch dann, wenn die Werbende einen Teil der Kosten der Leistung trage, ihre Leistung genauso wie bisher und zu demselben Entgelt erbrächten. Eine Zuwendung an die Ärzte liege danach schon nicht vor. Im Hinblick auf die Patienten läge zwar eine Zuwendung vor. Da es sich aber um einen bestimmten bzw. bestimmbaren Geldbetrag handele, sei diese Zuwendung zulässig. Ein Verstoß gegen \$7 HWG schied damit für das OLG aus. Zudem fehlte es nach dem Senat an einem Verstoß gegen $\$ 21$ Abs. 1 MBO-Z mangels berufsrechtswidriger Werbung. Insbesondere sei durch die Auslage der Flyer und der konkreten Werbung keine Nutzung der Berufsbezeichnung für gewerbliche Zwecke und auch keine Gestattung der Verwendung hierfür gegeben. Für einen Verstoß gegen das Gebot der eigenverantwortlichen und fachlich unabhängigen Ausübung der zahnärztlichen Tätigkeit bestanden keine Anhaltspunkte für den Senat. Daher schied auch auf dieser Basis eine Unlauterkeit aus.

\section{c) Werbung mit Erfolgsversprechen $\int 3 H W G$}

Wird ein Medizinprodukt (hier: Zahnspange) mit einer Wirkung beworben, die nicht vollständig objektivierbar ist, kann darin nach dem OLG Frankfurt a. M. ${ }^{12}$ dennoch ein unzulässiges Erfolgsversprechen liegen. Im $\mathrm{zu}$ entscheidenden Fall hatte die Beklagte ihre Zahnspange unter dem Hinweis angepriesen, damit ,,perfekte Zähne“ zu erhalten. Das OLG sah darin einen Verstoß gegen $\$ 3$ S. 1 Nr. 2a HWG und damit eine unlautere Handlung nach $₫ 3$ a UWG. Relevant für die Bewertung der Aussage sei, wie der Verkehr diese verstehe. Mit der Werbung für ,,perfekte Zähne" verspreche die Beklagte einen Behandlungserfolg. Dabei handele es sich, anders als von der Vorinstanz angenommen, auch nicht um ein rein subjektives Werturteil. Auch wenn die Frage, was ,,perfekte Zähne“ seien, nicht vollständig objektivierbar sei, enthalte die Werbung einen objektiven Tatsachenkern, der ein Erfolgsversprechen beinhalte, nämlich die Korrektur von Zahnfehlstellungen. Der angesprochene Verkehr sieht in dem Hinweis ,,perfekte Zähne" auch keine reklamehafte Übertreibung in Form eine Superlativwerbung. Insoweit spielte es nach dem Senat auch eine Rolle, dass die Werbung von Ärzten vorgenommen wurde, denen die Verbraucher besonderes Vertrauen entgegenbringen. Daher nehme der Verbraucher die Werbung im Zweifel ernst.

\section{d) Prozessrechtliches}

In zwei Entscheidungen, die ihren Ursprung in medizinprodukterechtlichen Streitigkeiten hatten (Schienen zur Zahnkorrektur; Mund-Nase-Schutzmasken), entschied das BVerfG über den Anspruch auf prozessuale Waffengleich-

9) OLG Hamm, Urt. v. 22.9.2020 - I-4 U 38/20 -, PharmR 2021, $140 \mathrm{ff}$.

10) OLG Stuttgart, Urt. v. 6.8.2020 - 2 W 23/20 -, PharmR 2020, $706 \mathrm{ff}$.

11) OLG Hamburg, Beschl. v. 14.4.2020 - 3 W 17/20 -, GRURRR 2021, $40 \mathrm{ff}$.

12) OLG Frankfurt a.M., Urt. v. 27.2.2020 - 6 U 219/19 -, NJWRR 2020, $614 \mathrm{ff}$. 
heit und rechtliches Gehör ${ }^{13}$. Danach ist es auch im einstweiligen Rechtsschutz grundsätzlich erforderlich, dass die Antragsgegnerin in das Verfahren einbezogen wird, wenn zwar außergerichtlich abgemahnt wurde, aber der Verfügungsantrag nicht identisch zu dem Unterlassungsbegehren ist. In diesem Zusammenhang könne es auch eine Pflicht geben, die Antragsgegnerin über einen gerichtlichen Hinweis an die Antragstellerin in Kenntnis zu setzen. Allerdings gelte insoweit auch die Kerntheorie, wonach der Schutzumfang eines Unterlassungsgebots nicht nur die identischen Verletzungsfälle erfasst, sondern auch gleichwertige Verletzungsfälle, die kerngleich sind. Dies sei entsprechend zu berücksichtigen.

\section{Haftungsrecht}

Der EuGH ${ }^{14}$ hat auf die Vorlagefrage des OLG Frankfurt a. M. ${ }^{15}$ im Rahmen eines Produkthaftungsverfahrens um fehlerhafte Brustimplantate klargestellt, dass die Klägerin des Ausgangsverfahrens sich gegenüber der Versicherung des Herstellers nicht auf Art. 18 Abs. 1 AEUV (Verbot der Diskriminierung aus Gründen der Staatsangehörigkeit) berufen könne. Die Klägerin verlangte von der Haftpflichtversicherung des Herstellers Ersatz des Schadens, der ihr durch den Austausch der Brustimplantate entstanden war. Der Versicherungsvertrag sah jedoch eine Haftungsbeschränkung vor, die die geografische Reichweite des Versicherungsschutzes auf Frankreich beschränkte. Das OLG Frankfurt legte dem EuGH unter anderem die Frage vor, ob diese Haftungsbeschränkung gegen Art. 18 Abs. 1 AEUV verstoße. Der EuGH prüfte zuerst, ob Art. 18 Abs. 1 AEUV überhaupt auf den vorliegenden Fall Anwendung findet. Der EuGH verneinte dies. Hierfür sei zu prüfen, ob ein unionsrechtlicher Bezug vorliege. Es fehle schon an einem Bezug zur Freizügigkeit der Unionsbürger. Die Klägerin, eine Deutsche, habe sich die Implantate in Deutschland, dem Land, in dem sie wohnt, einsetzen lassen. Damit habe sie im konkreten Fall schon gar nicht von der ihr zustehenden Freizügigkeit Gebrauch gemacht. Die Klägerin kann sich auch nicht auf einen Verstoß gegen die Dienstleistungsfreiheit nach Art. 56 AEUV berufen, so der EuGH. In Frage käme allenfalls der Versicherungsvertrag, auf Basis dessen sie den Direktanspruch gegen die Versicherung geltend machte. Dieser sei jedoch zwischen französischen Unternehmen geschlossen worden. Die Klägerin sei keine Vertragspartei. Damit scheide auch diese Anspruchsgrundlage aus, da es an einem nötigen Bezug zur Dienstleistungsfreiheit fehle. Gleiches gelte letztlich für die Warenverkehrsfreiheit nach Art. 34 AEUV. Der Sachverhalt betreffe schon nicht den freien Warenverkehr als solchen, sondern Mängel der Ware, die Gegenstand des freien Warenverkehrs waren. Damit war auch die Anwendung von Art. 18 Abs. 1 AEUV nach dem EuGH zu verneinen.

Dem OLG Karlsruhe ${ }^{16}$ lag ein Fall zur Produkthaftung wegen erhöhtem Metallabrieb bei einer Großkopfprothese zur Entscheidung vor. Der Kläger machte geltend, dass der Metallabrieb in der Konussteckverbindung die Fehlerhaftigkeit der Prothese begründe. Der Senat entschied, dass die „berechtigten Sicherheitserwartungen“ hier in Anbetracht der betroffenen Risikogruppe der Patienten zu bestimmen sind. Im vorliegenden Fall waren dies ältere und gesundheitlich vorgeschädigte Patienten. Diese Personengruppe erwarte bei Hüftprothesen, dass die als feste Verbindungen konzipierten Prothesenteile auch fest blieben. Ferner erwarte sie, dass die Prothese keine gesundheitsschädlichen Mengen an Metallpartikel an den Körper abscheide und eine Gesundheitsgefährdung insoweit ausgeschlossen sei. Bei Hüftprothesen gebe es grundsätzlich einen technisch unvermeidbaren Abrieb an der Gleitpaarung. Damit rechne der Verkehr auch. Die Herstellerin hatte insoweit mit einem besonders geringen Abrieb an der Gleitpaarung geworben.
Dann, so der Senat, gehe der Verkehr aber davon aus, dass an anderer Stelle, wie hier der Konussteckverbindung, kein Abrieb in gesundheitsgefährdenden Maße entstehe. Der Abrieb an der Konussteckverbindung wäre bei korrekter Implantierung der Prothese auch weitgehend vermeidbar gewesen. Hierfür wäre ein Fügen des Konusadapters auf den Prothesenschaft durch einen Schlag mit hoher Kraft nötig gewesen. Die Operationsanleitung sah hingegen nur einen ,leichten Schlag" vor. Damit sei diese fehlerhaft so das OLG. Die Ersatzpflicht sei auch nicht wegen der CEZertifizierung ausgeschlossen. Der Senat sprach dem Kläger in der Folge Ersatz seines Schadens nach $\$ \$ 1$ Abs. 1 S. 1, 8 ProdhaftG zu.

\section{Recht der gesetzlichen Krankenversicherung}

\section{a) Anspruch des Versicherten auf Hilfsmittel nach SS $33 \mathrm{ff}$. SGB V}

Der Versichertenanspruch auf Hilfsmittel beinhaltet grundsätzlich keine doppelte Ausstattung. Eine Doppelversorgung liegt jedoch nicht vor, wenn das vorhandene Hilfsmittel die zu befriedigenden Bedürfnisse nicht deckt und eine Ergänzung notwendig ist. Ein solcher Fall lag dem LSG Berlin-Brandenburg ${ }^{17}$ zur Entscheidung vor. Ein Jugendlicher, der von Geburt an keine rechte Hand hatte, begehrte eine neue VariPlus Speed Hand zur Ergänzung der vorhandenen elektrischen Unterarmprothese. Die vorhandene Prothese mit 14 wählbare Griffarten ermöglichte ihm zwar alltägliche Handlungen, die ein präzises Steuern des Greifens voraussetzen, wie z.B. Einnahme von Mahlzeiten, Öffnen von Türen, Binden von Schuhen. Für Tätigkeiten, die eine größere Griffkraft voraussetzen, wie z. B. Gartenarbeiten und Radfahren, war sie dagegen nicht geeignet. Eine ergänzende Versorgung des Jugendlichen mit der VariPlus Speed Hand, die nur aus Daumen, Mittel- und Zeigefinger besteht und ein kräftiges und zugleich schnelles Zugreifen ermöglicht, sah das LSG als notwendig an. Der unmittelbare Behinderungsausgleich sei auf ein Gleichziehen mit Gleichaltrigen auszurichten, um die Isolation des Jugendlichen zu verhindern. Insoweit gehe es nicht nur um den Schulbesuch als Grundbedürfnis, sondern auch um die Teilnahme an der sonstigen und üblichen Lebensgestaltung Gleichaltriger als Bestandteil eines sozialen Lernprozesses. Dementsprechend wies das LSG die Berufung der beklagten Krankenkasse zurück.

Im Rahmen eines einstweiligen Verfahrens sah das LSG Nordrhein-Westfalen ${ }^{18}$ den Anspruch eines Versicherten auf Versorgung mit einem Medizinprodukt (konkret: Elektrosimulationsgerät „PhySys“), das nach der Produktbeschreibung des Herstellers nur zur Anwendung durch medizinische Fachkreise, z.B. Ärzte, Therapeuten, Angehörige medizinischer Hilfsberufe vorgesehen ist, als nicht überwiegend wahrscheinlich an. Der Antragsteller war zwar Sanitätssoldat a. D. und Rettungssanitäter. Diese Qualifikationen sah das LSG jedoch als nicht ausreichend für die Anwendung der Elektro- und Ultraschalltherapie an, so dass es den Antragsteller nicht zum vorgesehenen Adressatenkreis zählte. Da der Hersteller zudem die Beobachtung des Patienten für notwendig erachtete, kam das

13) BVerfG, Beschl. v. 27.7.2020 - 1 BvR 1379/20 -, NJW 2020 $3023 \mathrm{ff}$;; BVerfG, Beschl v. 22.1.2021 - 1 BvR 2793/20 -, BeckRS 2021, 2602.

14) EuGH, Urt. v. 11. 6.2020 - C-581/18 -, BeckRS 2020, 11911

15) Vorjahresbericht Hobusch/Ochs, MedR 2019, 865 ff., sub 4.

16) OLG Karlsruhe, Urt. v. 8.6.2020 - 14 U 171/18 -, MDR 2020 , $990 \mathrm{ff}$.

17) LSG Bln.-Brdbg., Beschl. v. 11.11.2020 - L 28 KR 34/20 juris.

18) LSG Nordrh.-Westf., Beschl. v. 10.9.2020 - L 11 KR 362/20 B ER -, juris. 
LSG zu dem Schluss, dass die geplante häusliche Eigenanwendung des Medizinprodukts nicht geeignet und erforderlich sei, den Erfolg der Krankenbehandlung zu sichern, so dass das LSG einen Anordnungsanspruch verneinte und die Beschwerde des Versicherten gegen den erstinstanzlichen Beschluss zurückwies.

\section{b) Hilfsmitteleinsatz im Rahmen einer neuen Untersuchungs- und Behandlungsmethode}

Der Gemeinsame Bundesausschuss (G-BA) beschloss 2017 eine Richtlinie ${ }^{19}$ zur Erprobung der Transkorneale Elektrostimulation zur Behandlung der Retinitis Pigmentosa (erblich bedingte Erkrankung der Netzhaut). Obwohl das Erprobungsverfahren noch nicht abgeschlossen war und somit die Anerkennung dieser neuen Methode durch den GBA fehlte, bejahte das LSG Baden-Württemberg, wie in der letzten Rechtsprechungsübersicht erwähnt ${ }^{20}$, den Anspruch der klagenden Versicherten auf eine Versorgung mit dem bei der Behandlungsmethode zum Einsatz kommenden OkuStim ${ }^{\circledR}$ System (bestehend aus Gerät, Brille und Elektroden). Das LSG sah im Fall einer klagenden Versicherten einen Ausnahmefall gem. \2 Abs. 1a SGB V als gegeben an. Die Beschwerde der Beklagten gegen die Nichtzulassung der Revision verwarf das BSG mangels Darlegung einer Rechtsprechungsdivergenz als unzulässig ${ }^{21}$.

\section{c) Hilfsmittelverzeichnis $\int 139$ SGB V}

Die letzte Rechtsprechungsübersicht zum Medizinprodukterecht beinhaltete den Hinweis auf die Entscheidung des LSG Berlin-Brandenburg, nach der die klagende Herstellerin keinen Anspruch auf Aufnahme ihrer Spezialhausschuhe für Diabetiker in das Hilfsmittelverzeichnis hat, weil die Herstellerin den Nachweis der Funktionstauglichkeit und
Sicherheit der streitigen Hausschuhe nicht erbracht hatte ${ }^{22}$. Die Nichtzulassungsbeschwerde der Klägerin verwarf das BSG wegen nicht ausreichender Darlegung eines Zulassungsgrundes als unzulässig ${ }^{23}$.

In einem einstweiligen Verfahren vor dem SG München ${ }^{24}$ und Bay. $\mathrm{LSG}^{25}$ ging es um das im Hilfsmittelverzeichnis gelistete PleurX Peritoneal-Kathetersystem, das außerhalb des vorgesehenen Indikationsbereichs zum Einsatz kommen sollte. Lt. Hilfsmittelverzeichnis ist das Kathetersystem für die Pleuraerguss-Drainage bei moribunden Patienten mit Pleuracarcinose indiziert. Beim Antragsteller sollte es zur Behandlung einer pulmonalen Hypertonie mit stauungsbedingter Leberzirrhose und Aszites eingesetzt werden. Das SG führte aus, dass es sich beim Einsatz eines Produkts in einem nicht im Hilfsmittelverzeichnis enthaltenen Indikationsbereich, um einen Einsatz im Rahmen einer neuen Behandlungsmethode handele. Für diese neue Methode fehle die Anerkennung durch den G-BA, so dass der Antragsteller keinen Anspruch auf das Kathetersystem habe. Die gegen den Beschluss des SG gerichtete Beschwerde des Antragstellers wies das LSG zurück und begründete seine Entscheidung zudem mit vorhandenen Behandlungsalternativen.

19) Erprobungs-Richtlinie TES-RP vom 20.7.2017, BAnz AT 6.10.2017 B3, https://www.g-ba.de/richtlinien/99/.

20) LSG Bad.-Württ., Urt. v. 18.2.2020 - L 11 KR 2478/19 -, juris; Vorjahresbericht Hobusch/Ochs, MedR 2020, 911 ff., sub 5. b).

21) BSG, Beschl. v. 27.10.2020 - B $3 \mathrm{KR} 18 / 20 \mathrm{~B}-$, juris.

22) Vorjahresbericht Hobusch/Ochs, MedR 2020, 911 ff., sub 5. c).

23) BSG, Urt. v. 13.1.2021 - B 3 KR 10/20 B -, juris.

24) SG München, Beschl. v. 19.3.2020 - S 19 KR 279/20 ER BeckRS 2020, 12629.

25) Bay. LSG, Beschl. v. 10.6.2020 - L 4 KR 150/20 B ER BeckRS 2020, 12627.

\section{Der Aufbau der Europäischen Gesundheitsunion - Lernen aus der Corona-Krise}

\section{Karin Henke}

\begin{abstract}
:
Der Beitrag setzt an der Tagung des ineges v. 22.3.21 zum Thema „Ein Jahr Corona: Welche Lehren zieht das Mehrebenensystem? Zur Krisenresilienz und Alltagstanglichkeit des Gesundheitswesens." an. Die Verfasserin beschäftigt sich mit der Frage, welche Lehren aus der Corona-Krise gezogen wurden und inwiefern diese in den Aufbau der Europäischen Gesundheitsunion eingeflossen sind. Dabei werden die Handlungs- und Koordinierungsprobleme des Gesundheitssystems auf europäischer Ebene aufgezeigt und den neuen Legislativprojekten gegenübergestellt.
\end{abstract}

Karin Henke, wissenschaftliche Mitarbeiterin am ineges Institut für Europäische Gesundheitspolitik und Sozialrecht, promoviert zum Thema „Möglichkeiten und Grenzen für Erstattungsansprüche von Liposuktionen bei Lipödem“ bei Doktormutter: Prof. Dr. iur Astrid Wallrabenstein, Richterin des BVerfG, Gebäude RuW (Recht und Wirtschaft), Raum 3.126, Theodor-W.-Adorno-Platz 4, 60629 Frankfurt am Main, Deutschland
Die deutsche Gesundheitspolitik fußt auf dem politisch geprägten Gesundheitswesen und versteht sich als politische Querschnittsaufgabe ${ }^{1}$. Nach Busch hat sich Gesundheitspolitik lediglich auf Bereiche des körperlichen und psychischen Wohlbefindens zu beschränken ${ }^{2}$, soziale Rahmenbedingungen bleiben dabei außen vor ${ }^{3}$. Die Gesundheitspolitik wurde stets als nationale Aufgabe gesehen, jegliches Handeln fußte auf dieser Sichtweise. Das Auftreten des COVID-19-Virus und dessen Verbreitung über Ländergrenzen und Kontinente hinweg erfordert eine neue Denk-

1) König, Gesundheit, Gesundheitspolitik und soziale Gerechtigkeit: Überlegungen zu einer gesundheits-zentrierten Gesundheitspolitik, Hamburg, 2011, S. $131 \mathrm{ff}$.; Rosenbrock/Gerlinger, Gesundheitspolitik, 3. Aufl. 2014, S. 15 f.

2) Berg, Gesundheitsschutz als Aufgabe der EU: Entwicklung, Kompetenzen, Perspektiven, 1997, S. $60 \mathrm{ff}$; Schmidt am Busch, Die Gesundheitssicherung im Mehrebenensystem, 2007, S. 9.

3) Wallrabenstein, in: Schlachter/Heinig, Europäisches Arbeits- und Sozialrecht, Enzyklopädie Europarecht, Band 8, 2. Aufl. 2020, §8 Gesundheitspolitik, Rdnr. 2. 